Research Paper

\title{
GALNT6 Promotes Tumorigenicity and Metastasis of Breast Cancer Cell via $\beta$-catenin/MUC1-C Signaling Pathway
}

\author{
Yingge Mao',2,4\#, Yuqi Zhang1,2\#, Sairong Fan ${ }^{1,2 \#, ~ L v a o ~ C h e n ~}{ }^{1,2}$, Lili Tang ${ }^{1,2}$, Xiaoming Chen ${ }^{1,2}$, Jianxin \\ $\mathrm{Lyu}^{2,3}$ \\ 1. Institute of Glycobiological Engineering, School of Laboratory Medicine and Life Sciences, Wenzhou Medical University, Wenzhou, Zhejiang, China \\ 2. Zhejiang Provincial Key Laboratory of Medical Genetics, Key Laboratory of Laboratory Medicine, Ministry of Education, China, School of Laboratory \\ Medicine and Life Sciences, Wenzhou Medical University, Wenzhou, Zhejiang, China \\ 3. Hangzhou Medical College, Hangzhou, Zhejiang, China \\ 4. Present address: The First Affiliated Hospital of Henan University \\ \#These authors contributed equally to this work.
}

$\square$ Corresponding author: Xiaoming Chen, Institute of Glycobiological Engineering/School of Laboratory Medicine and Life Sciences, Wenzhou Medical University, Wenzhou, Zhejiang, 325035, China. Tel: 086-577-86699651; Fax: 086-577-86689717; E-mail: xmc@wmu.edu.cn

(c) Ivyspring International Publisher. This is an open access article distributed under the terms of the Creative Commons Attribution (CC BY-NC) license (https://creativecommons.org/licenses/by-nc/4.0/). See http://ivyspring.com/terms for full terms and conditions.

Received: 2018.08.07; Accepted: 2018.11.02; Published: 2019.01.01

\begin{abstract}
Polypeptide N-acetylgalactosaminyl transferase-6 (GALNT6), a member of the N-acetyl-D-galactosamine transferase family, was shown to be over-expression in mammary cancer and could be used as a biomarker. However, its roles and underlying mechanisms in the pathogenesis of breast cancer are still unclear. In this study, we reported that GALNT6 was up-expression in breast cancer, and it was not associated with tumor stage. The expression level of GALNT6 and menopause status was associated with patient survival. Biological function results illustrated that knockdown of GALNT6 inhibited proliferation, migration and invasion of MDA-MB-231 cells, and increased cell apoptosis. Knockdown of GALNT6 in breast cancer cell attenuated the protein expression of PCNA, cyclin D1, C-myc and $\beta$-catenin, and increased the expression of E-cadherin, caspase 3 and cleaved PARPI. Cell fractionation assay showed that knockdown of GALNT6 reduced the levels of $\beta$-catenin and MUCI-C in nucleus. Simultaneously knockdown of GALNT6 and $\beta$-catenin significantly reduced the level of C-myc. Co-IP experiments indicated that GALNT6 interacted with MUC1-N, $\beta$-catenin interacting with $\mathrm{MUCl}-\mathrm{C}$ in breast cancer cells. Together, our study reveals that GALNT6 promotes tumorigenicity and metastasis through $\beta$-catenin/MUCl-C signaling pathway.
\end{abstract}

Key words: glycosyltransferase; GALNT6; proliferation; metastasis, $\beta$-catenin

\section{Introduction}

Mammary cancer, the most common cancer among women worldwide, is the leading cause of premature mortality from cancer in women and the most common cause of death from cancer in less development regions women [1,2]. Tumor invasion and metastasis are the most prominent problem in clinical treatment of breast cancer, and about $10-15 \%$ of patients with breast cancer develops distant metastases within 3 years after the initial detection of primary tumor [3]. The high mortality rate of breast cancer is in part due to lack of early detection of breast cancer marker and novel molecular-targeted drugs with higher efficacy and low risk of adverse reactions $[4,5]$. Therefore, it is especially important to exploit novel biomarker or molecular-targeted drugs for clinical management of mammary tumor with effective diagnosis or therapies.

Glycosylation, one of the most important posttranslational modifications of proteins, is involved in many physiological events. Accumulating evidences show that changes in glycosylation accompany the acquisition of cellular features necessary for tumor growth and progression [6]. Aberrant glycosylation, which is commonly found in many cancers, has been 
shown to have multiple effects on cell growth, invasion, metastasis and immune surveillance $[7,8]$. In mammary cancer, aberrant glycosylation is associated with a worse prognosis and shorter overall survival, and leads to a higher incidence of remote metastasis [9]. Aberrant glycosylation are predominantly caused by changes in the levels of various glycosyltransferases, which are responsible for the transfer of carbohydrate to molecules, like proteins and lipids. O-glycaosylation, one of the major types of protein glycosylation, is catalyzed by a large family of polypeptide $\mathrm{N}$-acetylgalactosaminyltransferases (GalNAc-Ts), which transfer GalNAc to either serine or threonine residues in proteins, and associated with cancer progression and metastasis [10]. Emerging evidence has suggested that the expression of GalNAc-Ts was markedly upregulated in cancer cells, including breast cancer cells [11-12]. Polypeptide $\mathrm{N}$-acetylgalactosaminyltransferase-6 (GALNT6), one of the enzymes responsible for the initial step in O-glycosylation, was upregulated in various cancers, such as breast cancer, ovarian cancer, and pancreatic cancer [13-15]. Park et al [5] demonstrated that GALNT6 is correlated with the occurrence of lymph node metastasis, local recurrence and remote metastasis, and indicated that GALNT6 modifies Mucin 1 (MUC1) glycosylation and regulates proliferation of breast cancer cells. Prior studies have revealed that GALNT6 is upregulated in breast cancer cells, and might be a promising therapeutic target for inhibitors in therapeutic modalities against breast cancer in the future [16-18]. However, the mechanisms of GALNT6 in breast tumorigenicity are still not well understood.

In this study, we sought to better characterize the role of GALNT6 in breast cancer and understand of the mechanism of GALNT6 in breast tumourigenesis and metastasis. The level of GALNT6 was overexpressed in breast carcinoma, but was not correlated with patient survival. Subsequently, knockdown of GALNT6 was proved to inhibited cell growth, migration and invasion in a breast cancer cell line. Furthermore, GALNT6 enhances malignant growth of breast cancer cells appeared to be mediated by $\beta$-catenin/ MUC1-C signaling pathway. This study reveals that GALNT6 plays an important role in breast tumourigenesis and metastasis, suggesting that GALNT6 might be a potential treatment target against breast cancer.

\section{Results}

\section{GALNT6 is overexpressed in breast cancers and associated with patient survival in menopause status}

First, we examined expression levels of GALNT6 in breast cancers through publicly available TCGA data. Data retrieved from UALCAN web-portal [19] showed that GALNT6 was upregulated in breast carcinoma compared with normal breast tissue (Figure 1A). To further determine the association of GALNT6 in breast cancer, we analyzed the GALNT6 expression in the different stage in breast cancers. However, no significant differences in GALNT6 expression were observed with respect to tumor stage when the patients were stratified based on AJCC (American Joint Committee on Cancer) pathologic tumor stage (Figure 1B).

Survival analysis indicated that high GALNT6 expression was associated with poor overall survival (OS) (Figure 1C), however, there are no significant differentiation. Breast cancer involves various histopathological features known to have treatment implications, and can be subdivided into "HER2 positive", "Luminal" and "TNBC" groups [19]. Kaplan Meier analysis indicated that the high expression of GALNT6 in HER2 positive and TNBC groups have lower survival probability, compared with that in the low/medium expression of GALNT6 groups. The expression of GALNT6 has no effect on the survival probability in luminal group (Figure 1E). Additionally, Kaplan Meier analysis indicated that the GALNT6 expression and menopause status was significantly associated with survival probability (Figure $1 \mathrm{~F}, p=0.0012$ ). In peri-menopause and post-menopause status, the high expression of GALNT6 has higher survival probability. However, in pre-menopause status, the high expression of GALNT6 has the poorer survival.

\section{Down-regulation of GALNT6 inhibits breast cancer cell growth and promotes cell apoptosis in vitro}

To verify the GALNT6 expression in breast cancer cell lines, western blotting was performed in 3 human breast cancer cell lines and 1 non-tumorigenic epithelial cell line MCF-10. The results showed that the expression of GALNT6 was upregulated in the breast cancer cell lines (BT549, MDA-MB-231 and MCF-7), especially in breast cancer cell line MCF-7, compared with that in MCF-10 (Figure 2A). To examine the role of GALNT6 in tumor development in vitro, we generated the shRNA-T6 cell line by vector-based transfection of the sh-GALNT6 plasmid to inhibit GALNT6 expression in the MDA-MB-231 cell line, and a scrambled vector (shRNA-NC) was used as control. GALNT6 expression was significantly reduced in shRNA-T6 cells, compared with control cells (Mock and shRNA-NC), as assessed by qPCR (Figure 2B) and western blotting (Figure 2C). We next examined the effect of the down-regulation of GALNT6 on cell growth using the CCK8 assay and 
plate clone formation assay. As shown in Figure 2D, compared with the control cells (shRNA-NC), GALNT6 stable knockdown cells (shRNA-T6) showed significantly increased the cell growth inhibition rate. Similarly, the number of cell colonies in monolayer cultures of shRNA-T6 cells was significantly decreased, compared with that in control cells (Mock and shRNA-NC). Meanwhile, there was no significant difference between Mock and shRNA-NC group (Figure 2E). To further insight into the mechanism and investigate the protein involved in the cell proliferation, we examined the effect of GALNT6 on the expression of PCNA and cyclin D1. PCNA and cyclin D1 are two important protein associated with cell proliferation [20-21]. Western blotting analysis demonstrated that both the PCNA and cyclin D1 protein levels of cells transfected with sh-GALNT6 were markedly reduced compared with those of cells transfected with empty vector (shRNA-NC) or none (Mock) (Figure 4C and 4D). There were no significant differences in PCNA and cyclin D1 protein expression between Mock and shRNA-NC cells.
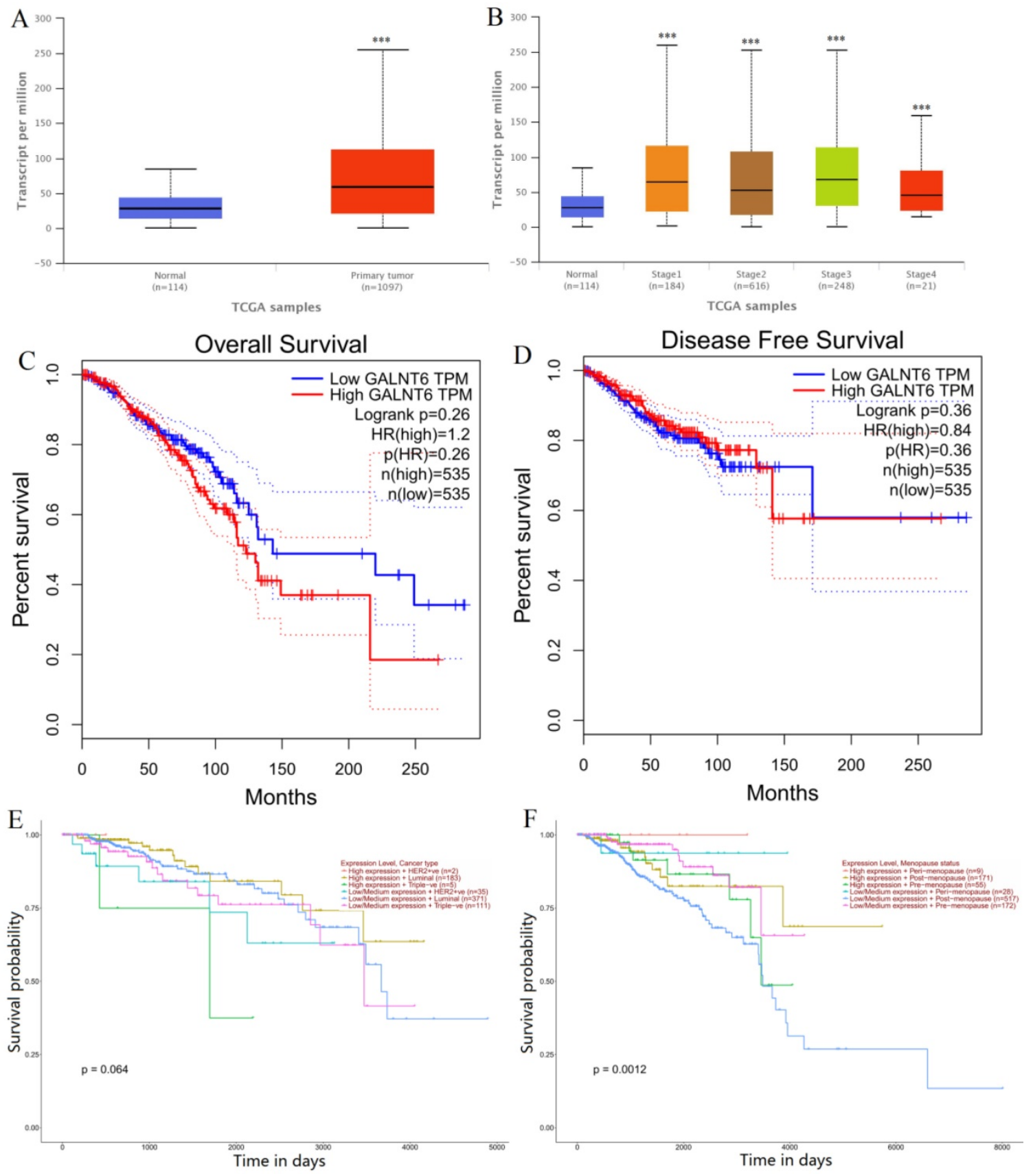

Figure 1. Relationships between GALNT6 expression and clinical features with patent survival. (A) Boxplot showing relative expression of GALNT6 in normal and breast carcinoma samples. (B) Boxplot showing relative expression of GALNT6 in normal and stage 1-4 breast cancer patients. (C) KM plot depicting association of GALNT6 expression levels with patient overall survival. (D) KM plot depicting association of GALNT6 expression levels with disease free survival. (E) KM plot depicting association of GALNT6 expression level and breast cancer subtype with patient survival. (F) KM plot depicting association of GALNT6 expression levels and menopause status with patient survival. 

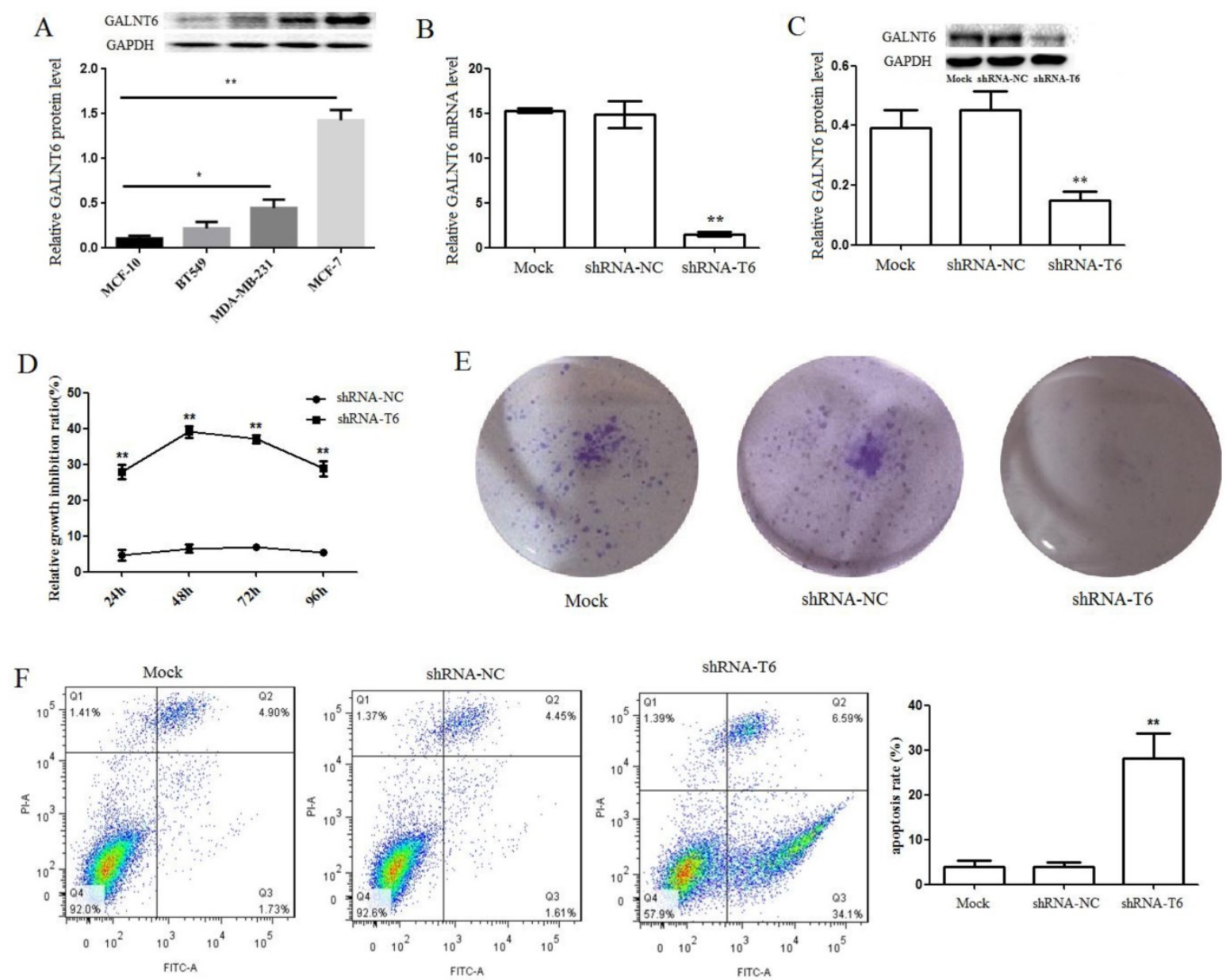

Figure 2. Down-regulation of GALNT6 inhibits MDA-MB-231 breast cancer cell proliferation and promotes cell apoptosis in vitro. (A) GALNT6 expression levels in breast cancer cell lines were detected by western blotting and quantified using Image software. (B) GALNT6 mRNA expression was quantified by qPCR. GALNT6 expression levels in shRNA-T6 and control (shRNA-NC and Mock) cells are shown. GAPDH expression was used for normalization. (C) GALNT6 expression was examined by western blotting analysis and quantified using Imagel software. The relative GALNT6 protein expression levels are shown. (D) CCK-8 cell assays in MDA-MB-231 cells. GALNT6 knockdown significantly inhibited the cell proliferative, compared to the controls. (E) The cloning ability was determined by colony formation assay in MDA-MB-231 cells. Compared with Mock and shRNA-NC cells, the colony formation was dramatically inhibited in shRNA-T6 cells. (F) The flow cytometry analysis cell apoptosis in MDA-MB-231 cells. Compared with Mock and shRNA-NC cells, the percentage of apoptotic cells was dramatically increased in shRNA-T6 cells. Data are expressed as means \pm SEM. $* P<0.05, * * P<0.01$.

Furthermore, the flow cytometry analysis revealed that the percentage of apoptotic cells significantly increased when GALNT6 was knocked down. There were $28.05 \pm 5.60 \%$ apoptotic cells in shRNA-T6 group, whereas in Mock and shRNA-NC groups, $4.03 \pm 1.26 \%$ and $3.90 \pm 1.11 \%$ apoptotic cells, respectively, was detected (Figure $2 \mathrm{~F}$ ). The apoptosis rate of shRNA-T6 cells was increased by 7-fold, compared with control cells. Bcl-2, which plays an important role in promoting cell survival and inhibiting cell apoptosis, was detected by western blotting. Compared with Mock and shRNA-NC cells, the level of $\mathrm{Bcl}-2$ was significantly reduced in shRNA-T6 cells (Figure 4E). Meanwhile, the apoptosis markers, such as cleaved poly(ADP-ribose) polymerase 1 (PARP1) and caspase-3, was detected. Compared with Mock and shRNA-NC cells, the shRNA-T6 cells showed higher level of caspase-3 and cleaved PARP1 (Figure 4G and $4 \mathrm{H}$ ), indicated that knockdown of GALNT6 promotes cell apoptosis, which was consistent with the flow cytometry analysis results. In addition, C-myc, which plays a very important role in regulating cell growth and apoptosis, was also analyzed by western blotting. Compared with Mock and shRNA-NC cells, the level of C-myc was dramatically reduced in shRNA-T6 cells (Figure 4F). These results suggested that down-regulation of GALNT6 may prevent breast cancer progression by suppressing cell growth and promotes cell apoptosis.

\section{Down-regulation of GALNT6 suppresses cell migration and invasion in vitro}

To characterize the effect of GALNT6 on cell migration and invasion, wound healing and Transwell assay were performed. Wound healing assay showed that, knockdown of GALNT6 resulted in a significant inhibition of wound closure, compared 
with control cells (Mock and shRNA-NC) (Figure 3A). Transwell migration assay showed that the number of cells having migrated through the filter was dramatically reduced in shRNA-T6 cells, compared with control cells (Mock and shRNA-NC) (Figure 3B). Consistent with the result of wound healing assay, down-regulation of GALNT6 decreased cell migration. In addition, matrigel invasion assay showed that cell invasion was also markedly suppressed in MDA-MB-231 cell with GALNT6 knockdown (Figure 3C). To further characterize the influence of GALNT6 on cell migration, we performed western blotting analysis to detect the expression levels of E-cadherin, which is known to be an inhibitor of tumor migration and invasion by the promotion of cell-cell interactions [22]. In breast cancer, partial or total loss of E-cadherin expression correlates with acquisition of invasiveness, increased metastatic behavior and poor prognoses [23]. Results showed that the expression level of E-cadherin was markedly increased in shRNA-T6 cells, compared with Mock and shRNA-NC cells (Figure 4A). These data revealed that downregulation of GALNT6 reduced motility and invasion of breast cancer cells in vitro.

\section{Down-regulation of GALNT6 suppresses breast cancer metastasis in vivo}

To further assess whether the down-regulation of GALNT6 affects the metastatic ability of MDA-MB-231 cells in vivo, we performed a lung metastasis experiment involving the inoculation of nude mice with Mock, shRNA-NC and shRNA-T6 cells via the venous plexus of the eye. Results showed that knockdown of GALNT6 significantly abrogated the ability of MDA-MB-231 cells to form metastatic foci in the lungs of nude mice (Figure 3D and 3E). These results indicated that knockdown of GALNT6 inhibited breast cancer metastasis in vivo.

\section{GALNT6 regulates $\beta$-catenin expression and distribution in breast cancer cells}

We found that knockdown of GALNT6 inhibited the expression of cyclinD1 and C-myc, two wellknown target genes of the Wnt/ $\beta$-catenin signaling pathway, which is broadly involved in the process of promoting tumorigenicity [24]. To gain insights into the possible molecular mechanism in breast cancer in response to the overexpression of GALNT6, we detected the expression of $\beta$-catenin, a key regulator of the canonical Wnt signaling pathway. Results showed that knockdown of GALNT6 significantly reduced the expression of $\beta$-catenin in mRNA and protein levels (Figure 4B). In addition, cell fractionation assay was performed to analyze the effects of GALNT6 expression on $\beta$-catenin distribution. The results showed that knockdown of GALNT6 in MDA-MB-231 cells suppressed $\beta$-catenin translocation into the nucleus and increased the membrane fraction of $\beta$-catenin, compared to control cells (Mock and shRNA-NC) (Figure 5A, left and right), indicating that GALNT6 regulates $\beta$-catenin translocation into the nucleus. Meanwhile, the cytoplasm fraction of $\beta$-catenin was slightly increased in shRNA-T6 cells compared with that in control cells (Mock and shRNA-NC) (Figure 5A, middle). To further confirm, the level of $\beta$-catenin in nucleus was monitored by confocal laser scanning microscope, and found the remarkable decrease of the signal intensity of nuclear $\beta$-catenin protein by knockdown of GALNT6 (Figure 6A).

\section{GALNT6 regulates $\beta$-catenin/MUC1-C signaling pathway in breast cancer cells}

To further demonstrate the influence of GALNT6 on $\beta$-catenin signal, cell fractionation assay was performed to analyze the effects of GALNT6 expression on MUC1-C, which is associated with $\beta$-catenin to translocate into the nucleus in triggering expression of oncogenes [25]. Results showed that knockdown of GALNT6 in MDA-MB-231 cells significantly reduced the expression of MUC1-C in cytoplasm and suppressed MUC1-C accumulation in the nucleus, compared to control cells (Mock and shRNA-NC) (Figure 5B), and the decrease of nuclear MUC1-C protein by knockdown of GALNT6 was further confirm by immunofluorescent assay (Figure $6 \mathrm{~B})$. Furthermore, to analyze the interaction of GALNT6, MUC1 and $\beta$-catenin, co-immunoprecipitation (Co-IP) assay was performed. The Co-IP assay demonstrated that GALNT6 and MUC1-N could precipitate down together, while $\beta$-catenin could precipitate with MUC1-C (Figure 5C), suggesting that GALNT6 interacted with MUC1-N, and $\beta$-catenin associated with MUC1-C. The results might suggest that GALNT6 is an activator to enhance the formation of the $\beta$-catenin/MUC1-C complex and translocation into the nucleus.

To ensure that GALNT6 regulate $\beta$-catenin signaling in breast cancer cells, we silenced $\beta$-catenin and investigated its activity. Knockdown of GALNT6 or $\beta$-catenin reduced the expression of C-myc, which is one of the target genes of $\beta$-catenin. The expression of C-myc was significantly reduced in simultaneously knockdown of GALNT6 and $\beta$-catenin cells, compared with that in knockdown of GALNT6 or $\beta$-catenin cells, indicating that GALNT6 regulated the expression of $\mathrm{C}$-myc via $\beta$-catenin signaling (Figure 7B). 


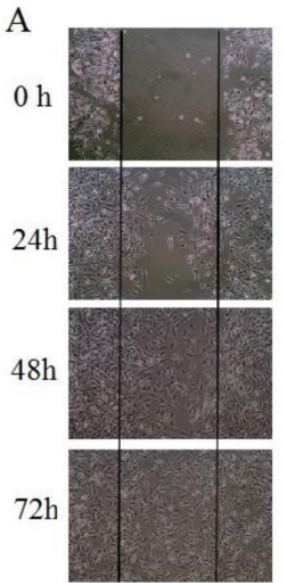

Mock
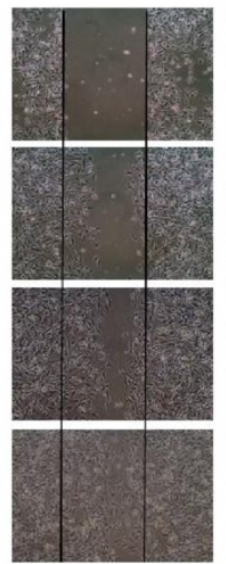

shRNA-NC
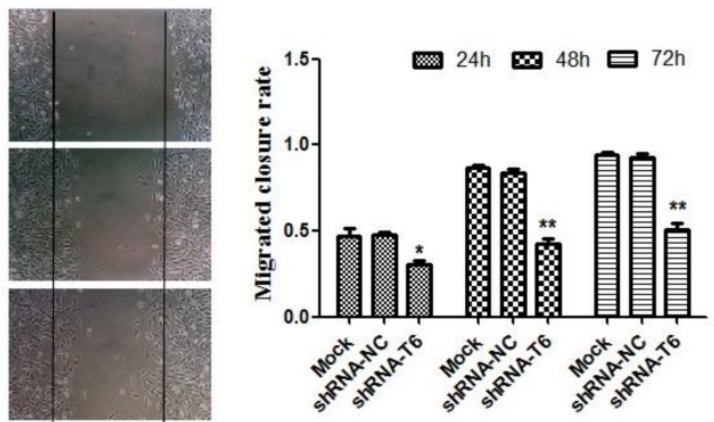

B

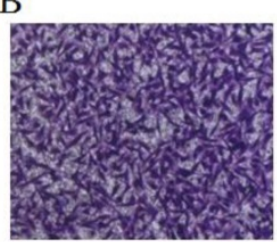

Mock

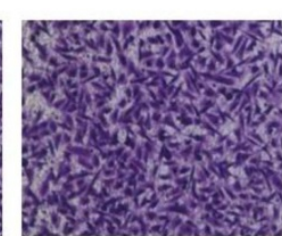

shRNA-NC

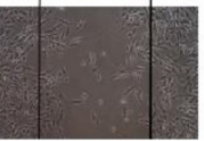

shRNA-T6

C

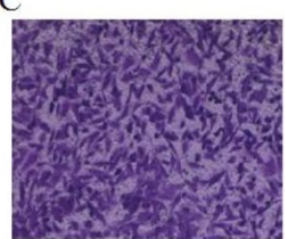

Mock

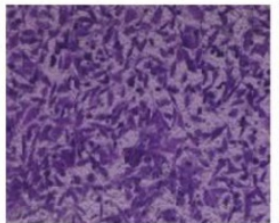

shRNA-NC

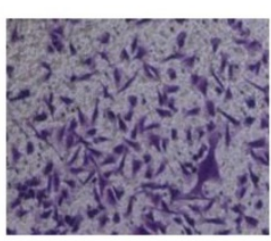

shRNA-T6

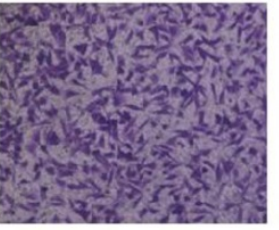

shRNA-T6
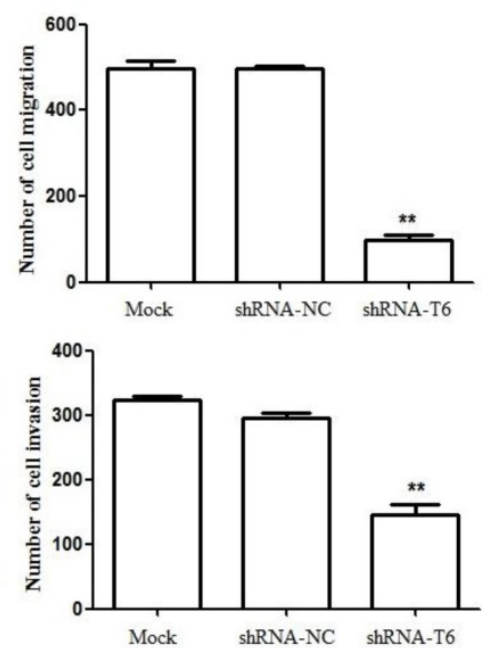

D
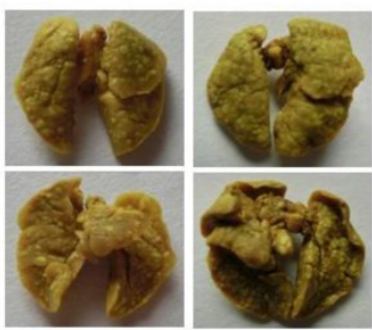

shRNA-NC
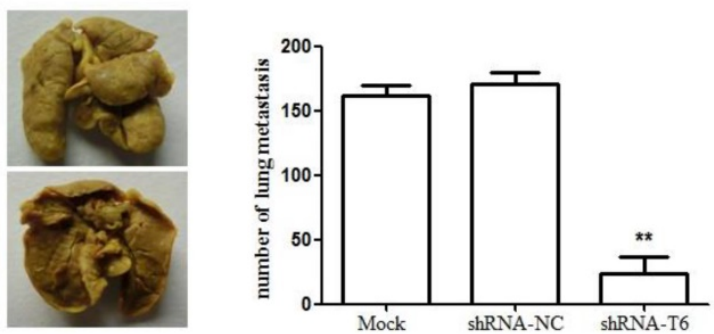

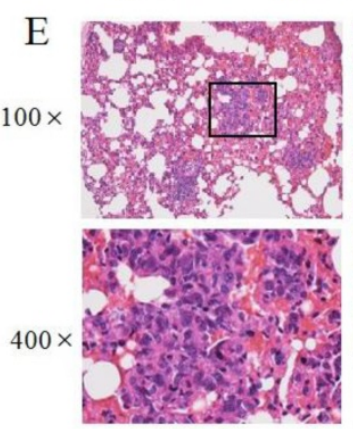

Mock

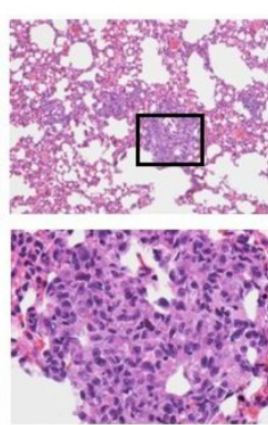

shRNA-NC

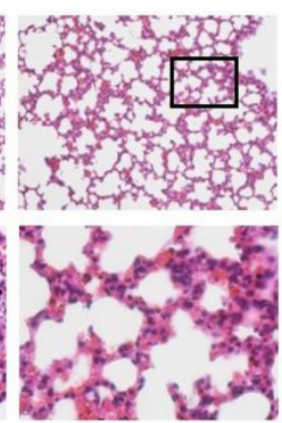

shRNA-T6

Figure 3. Down-regulation of GALNT6 inhibited the migration and invasion capacity of MDA-MB-231 cells. (A) Wound healing assay and quantification analysis showed that knockdown of GALNT6 significantly inhibited cell migration in MDA-MB-231 cells. (B) Transwell migration assay and quantification analysis showed that knockdown of GALNT6 significantly inhibited cell migration in MDA-MB-231 cells. (C) Matrigel invasion assay and quantification analysis showed that knockdown of GALNT6 greatly inhibited invasive abilities of MDA-MB-231 cells. (D-E) Nude mice $\left(n=5\right.$ per group) were injected with $1 \times 10^{7}$ MDA-MB-231 cells (Mock, shRNA-NC or shRNA-T6) via the venous plexus of the eye. After 4 weeks, the mice were sacrificed under anesthesia. (D) The lungs were subjected to Bouin's fixation and photographed. A representative of the experiments is shown. Visible lung metastases nudes were counted. (E) The sections of lungs were stained with H\&E. Data are expressed as means \pm SEM. $* P<0.05$ and $* * P<0.01$. 
A

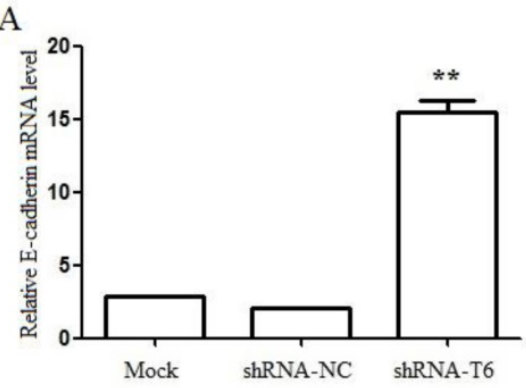

B

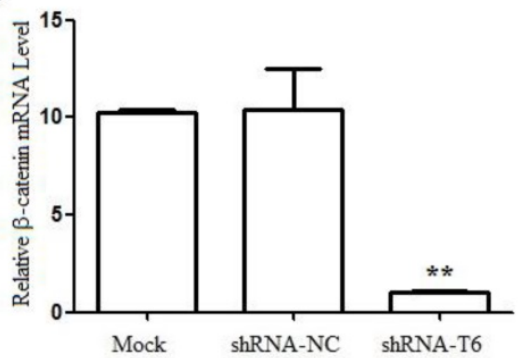

C

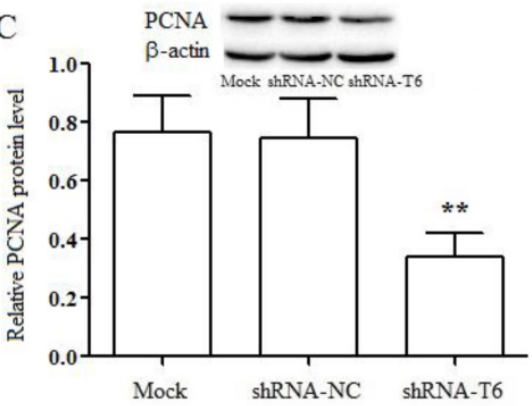

$\mathrm{E}$
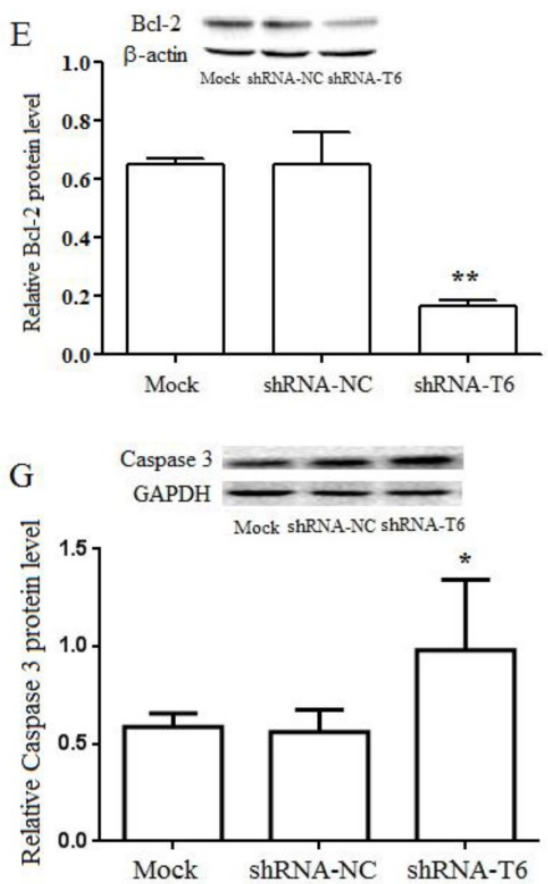

E-cadherin $=$
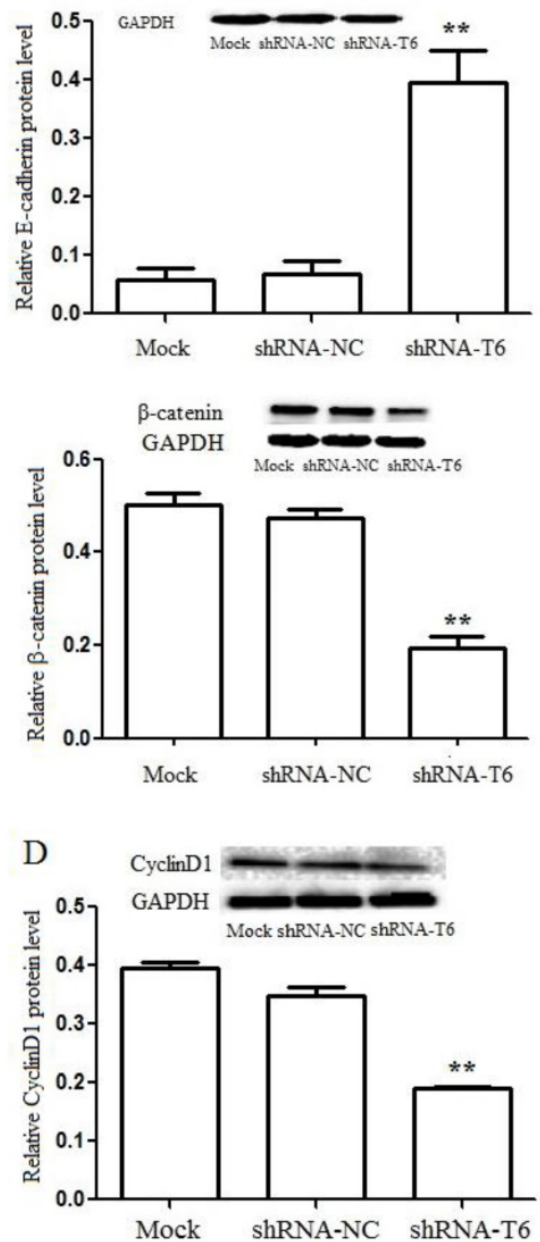

F
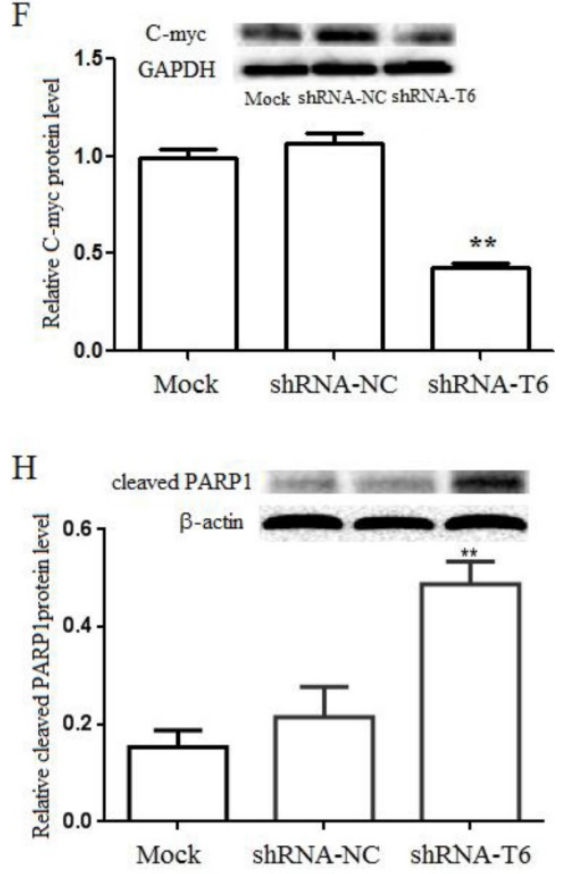

Figure 4. GALNT6 promotes MDA-MB-23 1 cells proliferation and migration through $\beta$-catenin signaling. (A) Effects of GALNT6 on the mRNA (left) and protein (right) expression of E-cadherin. Knockdown of GALNT6 increased the expression of E-cadherin in MDA-MB-231 cells. (B) Effects of GALNT6 on mRNA (left) and protein (right) the expression of $\beta$-catenin. Knockdown of GALNT6 decreased the expression of $\beta$-catenin in MDA-MB-231 cells. (C-H) Western blotting analysis. Compared with Mock and shRNA-NC cells, the levels of PCNA, cyclin DI, Bcl-2 and C-myc were significantly decreased in shRNA-T6 cells, while the levels of caspase- 3 and cleaved PARPI were significantly increased in shRNA-T6 cells. Data are expressed as means \pm SEM. $* P<0.05, * * P<0.01$. 


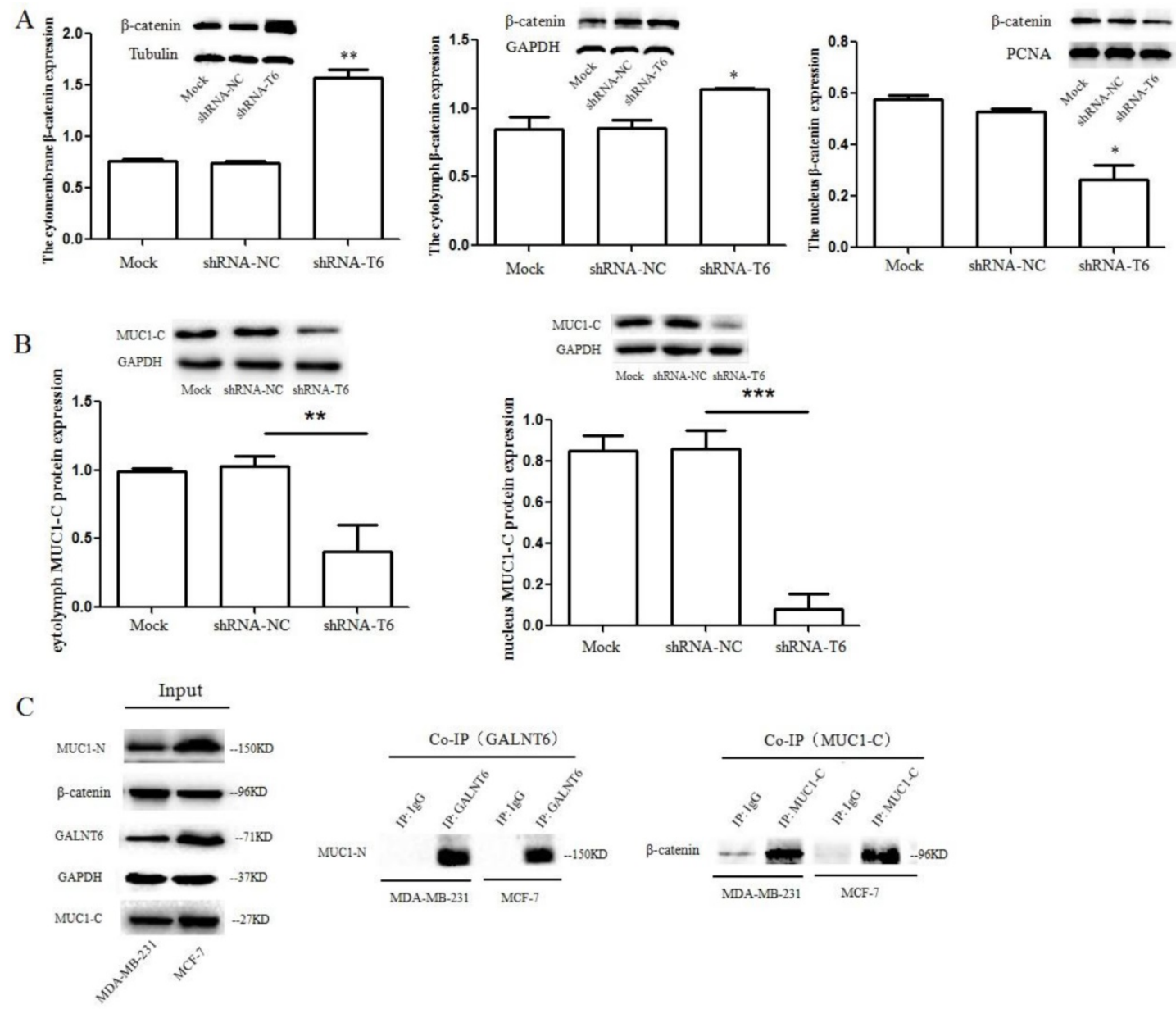

Figure 5. GALNT6 regulates $\beta$-catenin and MUCI-C distribution in MDA-MB-231 cells. (A) Effects of GALNT6 on the distribution of $\beta$-catenin. Knockdown of GALNT6 in MDA-MB-231 cells suppressed $\beta$-catenin translocation into the nucleus and increased the membrane fraction of $\beta$-catenin, compared with control cells (Mock and shRNA-NC). (B) Effects of GALNT6 on the expression and distribution of MUC1-C. Knockdown of GALNT6 in MDA-MB-231 cells reduced the expression of $\mathrm{MUCl}-\mathrm{C}$ in cytoplasm, and suppressed MUCl-C accumulation in the nucleus. (C) The interaction of GALNT6, MUCl and $\beta$-catenin in MDA-MB-231 and MCF-7 cells. Co-immunoprecipitation assays showed that knockdown of GALNT6 was association with MUC1-N, and $\beta$-catenin was association with MUCl-C. Data are expressed as means \pm SEM. $* P<0.05$, $* * P<0.01$ and $* * * P<0.001$.

\section{Discussion}

Glycosylation is a very important posttranslational modification of many biologically relevant molecules. Numerous reports have demonstrated an association between the aberrant expression of GalNAc-Ts family and cancer development. Accumulating evidences have shown that GALNT6 was upregulated in a number of types of cancer. In the present study, we used the publicly available TCGA data of GALNT6 in breast cancer by UALCAN and GEPIA web-portal, and analyzed the correlation between GALNT6 expression and survival probability. The expression of GALNT6 was upregulated in breast cancers, however, no correlation was observed between GALNT6 expression and tumor stage in breast cancers. The expression level of GALNT6 and menopause status was associated with patient survival.

Our previous studies have showed that GALNT6 can improve the proliferation and migration of MCF-7 cell [27]. To determine the exact function of GALNT6 in breast cancer, we used sh-GALNT6 against endogenously expression of GALNT6 in MDA-MB231 cells. Based on CCK8 and plate clone formation assay, we found that knockdown of GALNT6 significantly reduced the cell proliferation, which plays a very crucial role in the progression of cancer and metastatic progression of tumor cells in target microenvironments [28]. The levels of PCNA and cyclin D1, which are cell cycle related proteins and indicators of tumor proliferation and correlate with early cancer onset and tumor progression [29,30], were reduced in GALNT6 knockdown cells, indicating that GALNT6 might regulate PCNA and cyclin D1 expression promotes breast cancer cell proliferation. Flow cytometry assay showed that knockdown of GALNT6 significantly enhanced cell apoptosis, which was consistent with the WB results of apoptosis markers, such as Bcl-2, caspase 3 and cleaved PARP1. Meanwhile, the migration and 
invasion ability of MDA-MB-231 cells was decreased by GALNT6 knockdown. The promotion of tumor metastasis by GALNT6 was further confirmed in eye-venous plexus-injection metastatic mouse model. These results reveal that GALNT6 plays important role in the progression of breast cancer.

Membrane associated adhesion molecules mediate cellular adhesion, which are important for cell migration. E-cadherin, a transmembrane protein and known as the best-characterized molecular marker expressed in epithelial cell, plays a key role in cellular adhesion [25]. The loss of E-cadherin expression is positively correlated with the tumor stage, grade, and has been associated with greater tumor metastasis [31, 32]. The increased E-cadherin and membrane $\beta$-catenin in GALNT6 knockdown cells indicated that knockdown of GALNT6 not only promotes the expression of E-cdherin, but also increases the E-cdherin $/ \beta$-catenin complex, which would help to inhibit the cell migration.

$\beta$-catenin is related closely with tumourigenesis or metastasis, and is particularly interesting as it plays a dual role in the cell. First of all, it can act as a component of cell-cell adhesion complexes that helps cells maintain epithelial layers [33]. Secondly, $\beta$-catenin plays a key role in the Wnt signaling pathway. When Wnt is not present, cytoplasmic $\beta$-catenin protein is constantly degraded by the Axin complex, a complex was formed including phosphorylated $\beta$-catenin, APC, AXIN and GSK3 $\beta$, and following ubiquitination of $\beta$-catenin was degraded. When Wnt is present, $\beta$-catenin can't be phosphorylated, and it builds up in the cytoplasm and move into the nucleus to activate target genes, which lead to tumor development $[34,35]$. Transcriptionally active form of $\beta$-catenin induced readily multiple aggressive adenocarcinomas with overexpression of both c-Myc and cyclin D1 [36]. Here, the findings of western blotting and $\mathrm{QPCR}$ verified the downregulation of $\beta$-catenin in GALNT6 knockdown MDAMB-231 cells, and leaded to decrease the expression of cyclin D1 and C-myc, which are the Wnt signaling target genes. Meanwhile, simultaneously knockdown of GALNT6 and $\beta$-catenin significantly reduced the level of C-myc, indicating that GALNT6 might induce Wnt/ $\beta$-catenin signaling in MDA-MB-231 cell. Furthermore, cell fractionation assay demonstrated that GALNT6 regulated the distribution of $\beta$-catenin, and knockdown of GALNT6 suppressed $\beta$-catenin translocation into the nucleus. These results might suggest that GALNT6 mediated the activation of Wnt signaling pathways by regulation the expression and distribution of $\beta$-catenin, which activates target genes, such as cyclin D1 and C-myc, leading to cell proliferation and metastasis.
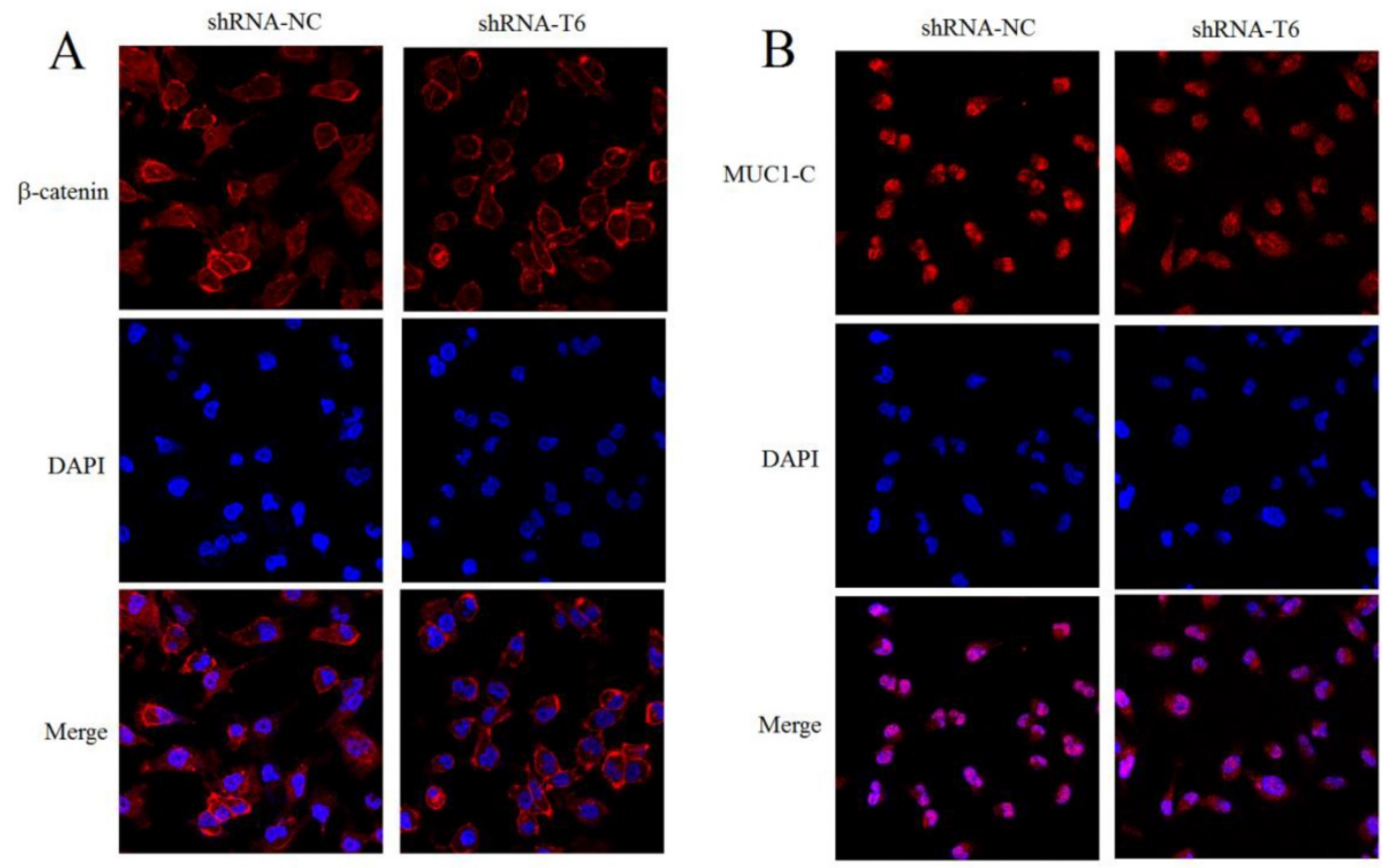

Figure 6. Knockdown of GALNT6 reduced $\boldsymbol{\beta}$-catenin and MUC1-C translocation into the nucleus. Immunofluorescent assay was performed in the shRNA-NC and shRNA-T6 stable cells. The $\beta$-catenin (A) and MUC1-C (B) present in the nucleus was monitored by measuring the blue fluorescence integrated over the nucleus, which was counterstained with DAPI. Knockdown of GALNT6 in MDA-MB-231 cells reduced the $\beta$-catenin and MUC1-C accumulation in the nucleus, compared with control cells (shRNA-NC). Results are representative of the three independent experiments. Magnification, $600 \times$. 
The details of how $\beta$-catenin shuttles between the cytoplasm and the nucleus are unclear. Reporters showed that MUC1-C facilitates $\beta$-catenin entry into the nucleus and activates the Wnt signaling pathway [37]. Mucin-1 (MUC1), a heterodimeric complex that includes N-terminal (MUC1-N) and C-terminal (MUC1-C) subunits, is one of the substrates of GALNT6, and which over-expression is hallmark of epithelial cancer [38,39]. In breast cancer cells, upregulation of GALNT6 could cause stabilization of MUC1 protein throughout its glycosylation activity [18]. Here, Co-IP assay confirmed that GALNT6 was interacted with MUC1-N, and $\beta$-catenin was interacted with MUC1-C. These results might suggest that GALNT6 not only increase the expression of MUC1, but also glycosylated MUC1 and released MUC1-C, which interacted with $\beta$-catenin and facilitates $\beta$-catenin entry into the nucleus. Knockdown of GALNT6 reduced the MUC1-C levels in cytoplasm and nucleus, which might attribute to the decrease the level of $\beta$-catenin in nucleus.

In conclusion, this study shows that GALNT6 expression is upregulated in breast cancer and attributed to tumor growth and metastasis in MDA-MB-231 cells by promoting cell proliferation, migration and invasion. We further elucidated the downstream signaling pathway responsible for GALNT6 oncogenic function in MDA-MB-231 cells and found that the GALNT6-mediated tumor growth and metastasis could be, at least partly, attributed to the activation of $\mathrm{Wnt} / \beta$-catenin/MUC1-C signaling pathway.

\section{Materials and Methods}

\section{Validation of the relationship between the expression of GALNT6 and clinical features of breast carcinomas in TCGA data}

The relationship between the expression of GALNT6 and clinical features of breast carcinomas was validated using the Cancer Genome Atlas (TCGA) data and analyzed by UALCAN (http:// ualcan.path.uab.edu) and GEPIA (http://gepia.can cer-pku.cn), which are newly developed interactive webs.

Based on AJCC (American Joint Committee on Cancer) pathologic tumor stage information, samples were divided into stage I, stage II, stage III and stage IV group. Primary breast cancer samples were divided into luminal, HER2 positive, and triple negative subclasses based on estrogen receptor (ER), progesterone receptor (PR), and human epidermal growth factor receptor 2 (HER2) status by immunohistochemistry (IHC). Patient data were categorized as pre-menopause, peri-menopause and post-menopause based on menopause status. Boxplots were generated of the expression level of gene across various subgroups. Multivariate Kaplan-Meier survival analysis was applied to assess the combined survival effect of gene expression and clinical parameters. The $P$ value obtained from log-rank test was used to indicate statistical significance of survival correlation between groups [19].

\section{Cell line and cell culture}

Human breast cancer cell line MDA-MB-231 ("basal" type and triple negative breast cancer cell line) and MCF-7 ("luminal" type and ER and PR positive breast cancer cell line), were purchased from American Type Culture Collection (ATCC). All of the cells were maintained in RPMI-1640 supplemented with 10\% FBS (Gibco, US) and 1\% penicillinstreptomycin (Gibco, US). All cell lines were incubited at $37^{\circ} \mathrm{C}$ in a humidified incubator with $5 \% \mathrm{CO}_{2}$.

\section{Establishment of the MDA-MB-231/ sh-GALNT6 and MDA-MB-231/Ctrl cell lines}

The shRNA-mediated gene silencing was carried out to construct GALNT6 knockdown cells using shRNA plasmids containing hairpin GALNT6 shRNA sequence. The pGPU6/GFP/Neo-GALNT6 interference vector and pGPU6/GFP/Neo-shNC control vector (Gemma, China) were transfected into MDA-MB-231 cells using Lipofectamine 3000 Transfection Reagent (Invitrogen) according to the manufacturer's instruction. Then, the clones were selected for 2 weeks under incubation with culture medium containing $0.9 \mathrm{mg} / \mathrm{ml}$ of geneticine (G418) (Gibco, USA). MDA-MB-231 cells stably expressing shNC (shRNA-NC) or shGALNT6 (shRNA-T6) were used in experiments. Mock (no insert) was used as negative control. The expression of GALNT6 in MDA-MB-231 was tested by Real-time Quantitative PCR and Western Blotting. The positive clones were selected for subsequent experiments.

\section{Cell proliferation assay}

Cell proliferation was analyzed using a Cell Counting Kit-8 (Dojindo, Japan). All cells were seeded in 96-well microplates at a density of 8000 cells per well and incubated at $37^{\circ} \mathrm{C}$ under $5 \% \mathrm{CO}_{2}$ for $24 \mathrm{~h}, 48$ $\mathrm{h}, 72 \mathrm{~h}$, or $96 \mathrm{~h}$, followed by the addition of $10 \mu \mathrm{l}$ Cell Counting Kit-8 (CCK8) reagent. The Absorbance in each well was measured at 1.5 hours using an automatic ELISA plate reader at a test wavelength of $450 \mathrm{~nm}$. Growth inhibition ratio $=\left(\mathrm{OD}_{0}-\mathrm{ODs}\right) / \mathrm{OD}_{0}$ $\times 100 \%, \mathrm{OD}_{0}$ : the OD at $450 \mathrm{~nm}$ of Mock, ODs: the OD at $450 \mathrm{~nm}$ of transfectants. Triplicate wells were set up in each group, and three independent experiments 
were performed.

\section{Colony formation assay}

Cells in the exponential phase of growth were digested and resuspended in complete medium. Then, the cells (Mock, shRNA-NC, or shRNA-T6) were seeded into 6-well plates containing $2 \mathrm{~mL}$ of medium at a density of 300 cells per well and incubated at $37^{\circ} \mathrm{C}$ under $5 \% \mathrm{CO}_{2}$. After 2 weeks, clone spheres had formed in the dishes. The cells were then fixed with $4 \%$ paraformaldehyde, and stained with crystal violet for $20 \mathrm{~min}$. The pictures were taken under microscope.

\section{Cell apoptosis analysis}

Cells were seeded into 6-well plates in RPMI-1640 containing 10\% FBS cultured for $48 \mathrm{~h}$. A total of $1 \times 10^{6}$ cells were harvested and double-stained with FITC-conjugated annexin $\mathrm{V}$ and Propidium Iodide (PI) using an Annexin V-FITC Apoptosis Detection Kit (Bio-Rad, USA), according to the manufacturer's protocol. The cells were assayed and quantified using the Flow Cytometer (Becton Dickinson, USA). All experiments were performed in triplicate and repeated three times.

\section{Wound healing assay and transwell migration assay}

Cell migration ability was evaluated by the wound healing assay and transwell chamber assay. Wound healing assay was performed to mimic cell migration. In brief, $1 \times 10^{6}$ cells were seeded into a 6-well plate. Two parallel straining lines were painted on each well. The wound width was measured every 24 hours over a period of 72 hours at $\times 100$ under Nikon Eclipse TS100 microscope (Nikon, Japan). Values were expressed as the percentage of wound closure, which was calculated as follows: percentage of wound closure $=1-\left(\right.$ width $_{t} /$ width $\left._{0}\right) \times 100 \%$.

Transwell migration assay was done in 24-well transwell chambers $(8 \mu \mathrm{m}$ pore diameter). Cells $\left(2 \times 10^{5}\right)$ were re-suspended in serum-free DMEM and added to the upper well of each migration chamber. Cell migration was induced by 10\% FBS in the lower chamber. After 24 hours, cells that migrated to the lower surface was fixed with $4 \%$ paraformaldehyde and stained with crystal violet for $15 \mathrm{~min}$ and counted. All experiments were performed in triplicate and repeated three times.

\section{Matrigel invasion assay}

Cell invasion assays were done in BioCoat Matrigel invasion chambers (Becton Dickinson, USA) according to the manufacture's protocol. Briefly, the upper chamber was pre-coated with BD Matrigel, then cells were added in the upper chamber with serum-free DMEM $\left(2 \times 10^{5}\right.$ cells/well $)$, and the lower chamber was loaded with standard culture medium. After 48 hours, the upper chamber was carefully swabbed with cotton, cells that has invaded through the membrane was fixed with $4 \%$ paraformaldehyde and stained with crystal violet. Cell numbers were counted for each well. All experiments were performed in triplicate and repeated three times.

\section{Quantitative real-time PCR (qPCR)}

Total RNA was extracted using TRIZOL reagent according to the manufacturer's protocol (Invitrogen), and cDNA was obtained by reverse transcription using a Takara reverse transcription kit (TaKaRa, China). The expression status of candidate genes and GAPDH were determined by quantitative real-time PCR using an ABI real-time fluorescent quantitative PCR system (Applied Biosystems, USA). Primers used were as follows: GALNT6: forward primer: 5'-AGAG AAATCCTTCGGTGACATT-3', reverse primer: 5'-AG

ACAAAGSGCCACAACTGAT
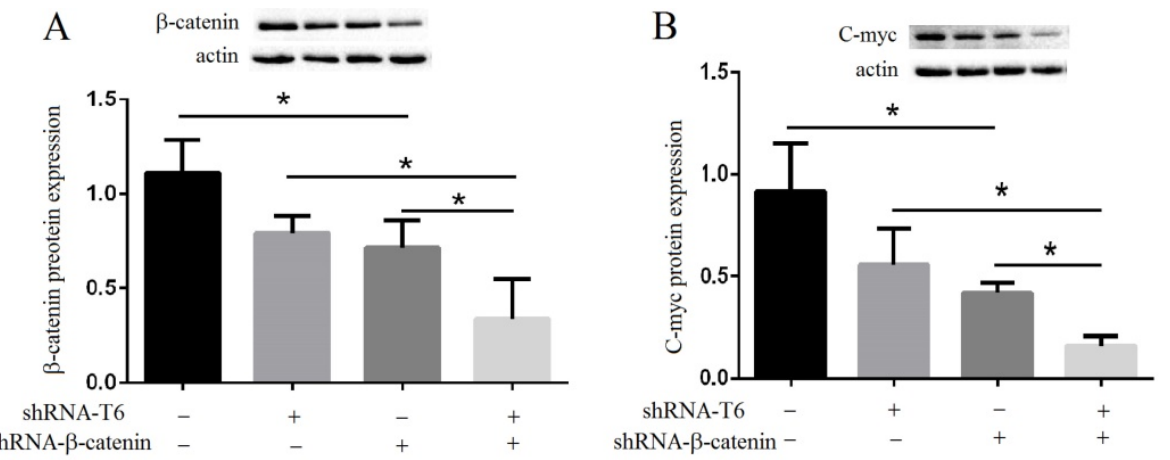

Figure 7. GALNT6 regulates $\mathbf{C}$-myc expression via $\boldsymbol{\beta}$-catenin signaling. (A) Western blotting and quantification analyses of the expression of $\beta$-catenin on GALNT6 or $\beta$-catenin knockdown in MDA-MB-231 cells. Knockdown of GALNT6 or $\beta$-catenin reduced the expression of $\beta$-catenin. (B) Western blotting and quantification analyses of the expression of C-myc on GALNT6 or $\beta$-catenin knockdown in MDA-MB-231 cells. The expression of C-myc was significantly reduced in simultaneously knockdown of GALNT6 and $\beta$-catenin cells, compared with that in knockdown of GALNT6 or $\beta$-catenin cells. Data are expressed as means \pm SEM. $* P<0.05$.
G-3'; E-cadherin: forward primer: 5'-GGGTTATTCCTCCCATC AGC-3', reverse primer: $5^{\prime}-\mathrm{GTC}$ ACCTTCAGCCATCCGT-3'; $\beta$ catenin: forward primer: $5^{\prime}-\mathrm{AG}$ GGTCTGGGACATTAGTCG-3', reverse primer: 5'-CGAAAGCC AATCAAACACAA-3'; GAPDH (as control): forward primer: 5'-GAACATCATCCCTGCCTCT ACT-3', reverse primer: 5'-CCTG CTTCACCACCTTCTTG-3'. All reactions were run in triplicate.

Western blotting analysis

Cells were lysed in RIPA 
buffer (Beyotime, China) supplemented with 0.1\% protease inhibitors (Sigma, USA), then centrifuged at $12,000 \mathrm{rpm}$ for $30 \mathrm{~min}$. The supernatant was collected and the protein concentration was quantified by BCA Protein Assay Kit (Beyotime, China). Equal amount of protein was separated on sodium dodecyl sulfate polyacrylamide gel and electrotransferred to PVDF membrane (Millipore Corp, US). The membrane was blocked by $5 \%$ skim milk and $0.1 \%$ Tween-20, followed by incubation with primary antibody overnight at $4{ }^{\circ} \mathrm{C}$. Primary antibodies were as following: anti-GALNT6, anti-CyclinD1, anti-MUC1-C and anti-GAPDH were purchased from Abcam (New Territories, USA), anti-E-cadherin, anti- $\beta$-catenin, anti-Bcl-2, anti-C-myc, anti-caspase 3, anti-cleaved PARP1 and anti- $\beta$-actin were purchased from Santa Cruz (CA, USA), anti-PCNA were purchased from Cell Signaling Technology (Danvers, USA). GAPDH and $\beta$-actin was served as a loading control. After incubating the primary antibody and second antibody, the bands were visualized with an enhanced chemiluminescence (ECL) system. These data were quantified by densitometry. The Image J software was analyzed the protein relative expression. All experiments were performed in triplicate.

\section{Immunoprecipitation}

Cell lysates were centrifuged $(12,000 \mathrm{rpm}, 20$ $\min , 4^{\circ} \mathrm{C}$ ) and the supernatant was pre-cleared with normal rabbit IgG and sepharose-protein A/G beads, and then incubated with corresponding antibody at 4 ${ }^{\circ} \mathrm{C}$ for $2 \mathrm{~h}$ on a rotator. Protein-antibody complexes were collected with pre-washed sepharose-protein A/G beads at $4^{\circ} \mathrm{C}$ overnight. The product was collected by centrifugation (3000 rpm, $5 \mathrm{~min})$, then washed three times with PBS, and finally resuspended in the SDS sample buffer. After being boiled at $100{ }^{\circ} \mathrm{C}$ for $10 \mathrm{~min}$, the immunoprecipitated complexes were released for western blotting analysis.

\section{Immunofluorescent assay}

Briefly, cells were seeded in 24-well plates and stained once they reached $60 \%$ confluence. Cells were fixed with $4 \%$ paraformaldehyde for $30 \mathrm{~min}$, and permeabilized with $0.5 \%$ Triton X-100 for $15 \mathrm{~min}$. After incubated with an anti- $\beta$-catenin (Santa Cruz, USA) or anti-MUC1-C (Santa Cruz, USA) primary antibody for $16 \mathrm{~h}$ at 4 degree, cells were incubated with the secondary antibody Cy3-labeled Goat AntiRabbit IgG (H+L) (Beyotime Biotechnology, China). Finally, cells were stained with $0.5 \mu \mathrm{g} / \mathrm{mL} \mathrm{4}$,6diamidino-2-phenylindole (DAPI) (Sigma-Aldrich, USA) and examined by confocal laser scanning microscope.

\section{Animal experiments}

BALB/c nude mice (6 week old, female) were provided by the Shanghai Laboratory Animal Center (Shanghai, China). Mice were maintained and subjected to experiments in accordance with the guidelines established by Institute for Experimental Animals of Wenzhou Medical University, and approved by the university committee for animal experiments. MDA-MB-231 cells stably expressing shNC (shRNA-NC) or shGALNT6 (shRNA-T6) were used in animal experiments. MDA-MB-231 cells (Mock) were used as negative control.

For the tumor metastasis assay, BALB/C-nu mice were injected with $1 \times 10^{7}$ cells per animal via the venous plexus of the eye ( 5 mice in each group). The mice were sacrificed after 4 weeks inoculation under anesthesia and the lung metastases were examined. The lung tissues were fixed in Bouin's solution and the number of metastatic nodules was counted.

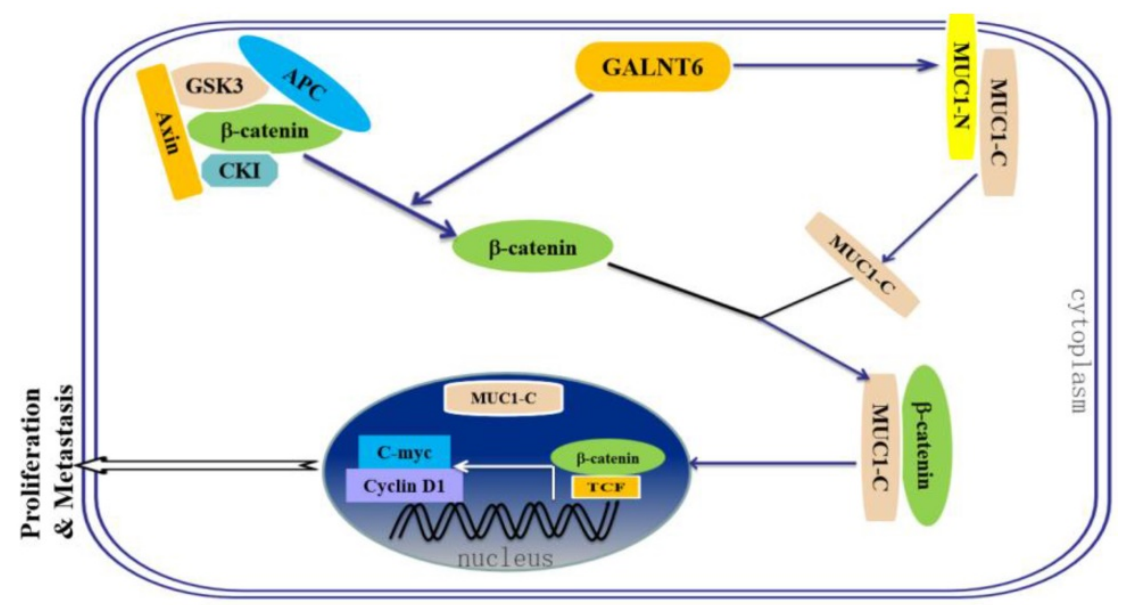

Figure 8. A proposed model of the mechanism of GALNT6-mediated regulation of proliferation and metastasis thought $\beta$-catenin /MUC1-C signaling in breast cancer. 


\section{Statistical analysis}

Statistical analyses were performed using SPSS software version 17.0 (Chicago, Illinois, USA). Survival curves were plotted by Kaplan-Meier method and compared by log-rank test. Student's t-test was used to analyze in vitro experiments in cell lines. If two groups could not be consider to be of equal variance, Dunnett's multiple comparison test was performed. Data was expressed as the mean value \pm SEM. $P$-values of less than 0.05 were considered to be statistically significant. All experiments were performed in triplicate.

\section{Abbreviations}

GALNT6: Polypeptide N-acetylgalactosaminyl transferase-6; GalNAc-Ts: polypeptide N-acetylgalactosaminyltransferases; TCGA: The Cancer Genome Atlas; AJCC: American Joint Committee on Cancer; ER: Estrogen receptor; PR: progesterone receptor; HER2: human epidermal growth factor receptor 2; TNBC: Triple negative breast cancer; IHC: Immunohistochemistry; G418: Geneticine; CCK8: Cell Counting Kit-8; PI: Propidium Iodide; qPCR: Quantitative real-time PCR; Co-IP: Co-immunoprecipitation; MUC1: Mucin 1; PARP1: Poly(ADP-ribose) polymerase 1; KM: Kaplan Meier; OS: Overall survival.

\section{Acknowledgments}

This work was supported by the National Natural Science Foundation of China (No. 31570805), and Key Discipline of Zhejiang Province in Medical Technology (First Class, Category A).

\section{Competing Interests}

The authors have declared that no competing interest exists.

\section{References}

1. Siegel RL, Miller KD, and Jemal A. Cancer statistics, 2018. CA Cancer J Clin. 2018; 68: 7-30.

2. Gonzaga C, Freitas-Junior R, Curado M, et al. Temporal trends in female breast cancer mortality in Brazil and correlations with social inequalities: ecological time-series study. BMC Public Health. 2015; 15: 1445.

3. Chou $\mathrm{CH}$, Huang MJ, Chen $\mathrm{CH}$, et al. Up-regulation of C1GALT1 promotes breast cancer cell growth through MUC1-C signaling pathway. Oncotarget. 2015; 6(8): 6123-6135.

4. Yang MD, Chang WS, Tsai CW, Wang MF, Chan YC, et al. Inhibitory Effects of AVEMAR on Proliferation and Metastasis of Oral Cancer Cells. Nutr Cancer. 2016; 68(3): 473-480

5. Park JH, Nishidate T, Kijima K, et al. Critical roles of mucin 1 glycosylation by transactivated polypeptide $\mathrm{N}$-acetylgalactosaminyltransferase 6 in mammary carcinogenesis. Cancer Res. 2010; 70: 2759-2769.

6. Magalhães A, Duarte H, and Reis C. Aberrant Glycosylation in Cancer: A Novel Molecular Mechanism Controlling Metastasis. Cancer Cell. 2017; 31: 733-735.

7. Gao Y, Liu Z, Feng J, et al. Expression pattern of polypeptide Nacetylgalactosaminyltransferase-10 in gastric carcinoma. Oncol Lett. 2013; 5: 113-116.

8. Guda $\mathrm{K}$, Moinova $\mathrm{H}, \mathrm{He} \mathrm{J}$, et al. Inactivating germ-line and somatic mutations in polypeptide $\mathrm{N}$-acetylgalactosaminyltransferase 12 in human colon cancers. Proc Natl Acad Sci USA. 2009; 106: 12921-12925.
9. Liesche F, Kölbl AC, Ilmer M, Hutter S, Jeschke U, and Andergassen U. Role of $\mathrm{N}$-acetylgalactosaminyltransferase 6 in early tumorigenesis and formation of metastasis. Mol Med Rep. 2016; 13(5): 4309-4314.

10. Schjoldager KT, and Clausen $\mathrm{H}$. Site-specific protein O-glycosylation modulates proprotein processing - deciphering specific functions of the large polypeptide GalNAc-transferase gene family. Biochim Biophys Acta. 2012; 1820: 2079-2094.

11. Brooks S, Carter T, Bennett E, Clausen $\mathrm{H}$, and Mandel U. Immunolocalisation of members of the polypeptide $\mathrm{N}$-acetylgalactosaminyl transferase (ppGalNAc-T) family is consistent with biologically relevant altered cell surface glycosylation in breast cancer. Acta Histochem. 2007; 109: 273-284.

12. Lin JY, Chung SY, Ueda K, Matsuda K, Nakamura Y, and Park JH. GALNT6 stabilizes GRP78 protein by O-glycosylation and enhances its activity to suppress apoptosis under stress condition. Neoplasia. 2017; 19: 43-53.

13. Gomes J, Marcos N, Berois N, et al. Expression of UDP-N-acetyl-Dgalactosamine: polypeptide $\mathrm{N}$-acetylgalactosaminyltransferase- 6 in gastric mucosa, intestinal metaplasia, and gastric carcinoma. J Histochem Cytochem. 2009; 57: 79-86

14. Vitiazeva V, Kattla JJ, Flowers SA, et al. The O-Linked Glycome and Blood Group Antigens ABO on Mucin-Type Glycoproteins in Mucinous and Serous Epithelial Ovarian Tumors. PLoS One. 2015; 10: e0130197.

15. Tarhan YE, Kato T, Jang M, et al. Morphological Changes, Cadherin Switching, and Growth Suppression in Pancreatic Cancer by GALNT6 Knockdown. Neoplasia. 2016; 18(5): 265-272.

16. Freire $\mathrm{T}$, Berois $\mathrm{N}$, Sóñora $\mathrm{C}$, et al. UDP-N-acetyl-D-galactosamine: polypeptide $\mathrm{N}$-acetylgalactosaminyltransferase 6 (ppGalNAc-T6) mRNA as a potential new marker for detection of bone marrow-disseminated breast cancer cells. Int J Cancer. 2006; 119: 1383-1388.

17. Patani N, Jiang W, and Mokbel K. Prognostic utility of glycosyltransferase expression in breast cancer. Cancer Genom Proteom. 2008; 5(6): 333-340.

18. Park J, Katagiri T, Chung S, Kijima K, and Nakamura Y. Polypeptide $\mathrm{N}$-acetylgalactosaminyltransferase 6 disrupts mammary acinar morphogenesis through O-glycosylation of fibronectin. Neoplasia. 2011; 13: 320-326.

19. Chandrashekar DS, Bashel B, Balasubramanya SAH, et al. UALCAN: A portal for facilitating tumor bubgroup gene expression and survival analyses. Neoplasia. 2017; 19: 649-658.

20. Kubben FJ, Peeters-Haesevoets A, Engels LG, et al. Proliferating cell nuclear antigen (PCNA): a new marker to study human colonic cell proliferation. Gut. 1994; 35: 530-535.

21. Baldin V, Lukas J, Marcote MJ, Pagano M, and Draetta G. Cyclin D1 is a nuclear protein required for cell cycle progression in G1. Genes Dev. 1993; 7: 812-821.

22. Brockhausen I. Mucin-type O-glycans in human colon and breast cancer: glycodynamics and functions. EMBO Rep. 2006; 7: 599-604.

23. Berx G, and Roy FV. The E-cadherin/catenin complex: an important gatekeeper in breast cancer tumorigenesis and malignant progression. Breast Cancer Res. 2001; 3: 289-293.

24. Yang SC, Liu Y, Li MY, et al. FOXP3 promotes tumor growth and metastasis by activating Wnt/ $\beta$-catenin signaling pathway and EMT in non-small cell lung cancer. Mol Cancer. 2017; 16(124): 1-12.

25. Kufe DW. MUC1-C oncoprotein as a target in breast cancer: activation of signaling pathways and therapeutic approaches. Oncogene. 2013; 32:1073-1081.

26. Banford S, and Timson DJ. UDP-N-acetyl-D-galactosamine: polypeptide $\mathrm{N}$-acetylgalactosaminyltransferase-6 (pp-GalNAc-T6): Role in Cancer and Prospects as a Drug Target. Curr Cancer Drug Tar. 2017; 17: 53-61.

27. Zhou WY, Ren J, Ji Y, and Chen XM. The roles of N-acetyl galactosyltransferase- 6 in proliferation and migration of breast cancer cell MCF-7. J. Wenzhou Medical University. 2017; 47(12): 864-868.

28. Zhang Q, Chen X, Zhang X, Zhan J, and Chen J. Knockdown of TMEM14A expression by RNAi inhibits the proliferation and invasion of human ovarian cancer cells. Biosci Rep. 2016; 36(1): e00298.

29. Bao Z, Duan C, Gong C, et al. Protein phosphatase $1 \gamma$ regulates the proliferation of human glioma via the NF-kappaB pathway. Oncol Rep. 2016; 35(5): 2916-2926.

30. Lehn S, Tobin NP, Berglund P, et al. Down-regulation of the oncogene cyclin D1 increases migratory capacity in breast cancer and is linked to unfavorable prognostic features. Am J Pathol. 2010; 177: 2886-2897.

31. Christiansen JJ, and Rajasekaran AK. Reassessing epithelial to mesenchymal transition as a prerequisite for carcinoma invasion and metastasis. Cancer Res. 2006; 66: 8319-8326.

32. Beavon IR. The E-cadherin-catenin complex in tumour metastasis: structure, function and regulation. Eur J Cancer. 2000; 36: 1607-1620.

33. Miyoshi $K$, and Hennighausen L. $\beta$-Catenin: a transforming actor on many stages. Breast Cancer Res. 2003; 5: 63-68.

34. Mosimann C, Hausmann G, and Basler K. $\beta$-catenin hits chromatin: regulation of Wnt target gene activation. Nat Rev Mol Cell Bio. 2009; 10(4): 276-286.

35. MacDonald BT, Tamai $\mathrm{K}$, and He X. Wnt/ $\beta$-catenin signaling: components, mechanisms, and diseases. Dev. Cell. 2009; 17(1): 9-26.

36. Imbert A, Eelkema R, Jordan S, Feiner $\mathrm{H}$, and Cowin P. DeltaN89beta-catenin induces precocious development, differentiation, and neoplasia in mammary gland. J Cell Biol. 2001; 153: 555-568. 
37. Zhang LL, Gallup M, Zlock L, et al. Pivotal role of MUC1 glycosylation by cigarette smoke in modulating disruption of airway adherens junctions in vitro. J Pathol. 2014; 234: 60-73

38. Remmers N, Anderson J, Linde E, et al. Aberrant expression of mucin core proteins and o-linked glycans associated with progression of pancreatic cancer. Clin Cancer Res. 2013; 19: 1981-1993.

39. Kim YS, Gum J, and Brockhausen I. Mucin glycoproteins in neoplasia. Glycoconj J. 1996; 13: 693-707. 